\title{
Differences in treatment response to a total diet replacement intervention versus a food-based intervention: A secondary analysis of the OPTIWIN trial
}

\author{
Jamy D. Ard ${ }^{1}$ (1) | Kristina H. Lewis ${ }^{1}$ @ | Sarah S. Cohen ${ }^{2}$ | Amy E. Rothberg ${ }^{3}$ | \\ Sally L. Coburn ${ }^{4}$ | Judy Loper ${ }^{5}$ | Laura Matarese ${ }^{6}$ | Walter J. Pories ${ }^{6}$ | \\ Seletha Periman ${ }^{7}$
}

${ }^{1}$ Department of Epidemiology and Prevention Wake Forest School of Medicine, WinstonSalem, North Carolina, USA

${ }^{2}$ EpidStrategies, Cary, North Carolina, USA

${ }^{3}$ Department of Internal Medicine, Michigan Medicine, Ann Arbor, Michigan, USA

${ }^{4}$ Alaska Premier Health, Anchorage, Alaska, USA

${ }^{5}$ The Central Ohio Nutrition Center, Inc., Gahanna, Ohio, USA

${ }^{6}$ Department of Surgery, East Carolina University Brody School of Medicine, Greenville, North Carolina, USA

${ }^{7}$ Nestlé Health Science, Global Research \& Development, Bridgewater, New Jersey, USA

\section{Correspondence}

Jamy D. Ard, Department of Epidemiology and Prevention, Wake Forest School of Medicine, Medical Center Boulevard, Winston-Salem, NC 27157, USA.

Email: jard@wakehealth.edu

[Correction added on 19 August 2020, after first online publication: Affiliation 2 was mistakenly associated with Amy E. Rothberg but instead is the affiliation for Sarah S. Cohen. This has now been corrected.]

\section{Summary}

Objective: For every weight loss treatment, there are usually groups of people who lose less than expected. This study sought to determine if response rates to a total diet replacement (TDR) differed from those of a calorie-restricted, food-based (FB) diet.

Methods: Data from OPTIWIN, a 12-month multicenter trial in adults with a BMI of $30-55 \mathrm{~kg} / \mathrm{m}^{2}$, with 26-week weight-loss and weight-maintenance phases, were utilized. Participants $(n=330)$ were randomized to the OPTIFAST programme (OP) or to a reduced-energy FB diet. Treatment non-responders were defined as those who lost $<3 \%$ of initial weight at months 6 or 12 .

Results: There were 103 (76\%) responders in the OP compared with 78 (57\%) in the FB group at 12 months. The odds of treatment response at 12 months among participants who were non-responders at 3 months was not significantly different between the OP and FB groups ( $p=0.64)$. Race, type 2 diabetes status and previous weight loss attempts were significantly associated with responder status. OP responders had higher meal plan adherence and non-caloric fluid intake compared with FB responders. Conclusion: Early treatment response is more likely and better sustained with TDR compared with an FB diet. Individual and treatment level factors appear to influence early treatment response to behavioural interventions for weight reduction.

KEYWORDS

behavioural weight loss, total diet replacement, treatment response

\section{$1 \mid$ INTRODUCTION}

Most weight reduction strategies will have a proportion of individuals who do not respond to treatment, with the definition of 'non-response' varying according to the expected weight loss for an intervention. For lifestyle and pharmacotherapy interventions, patients who have lost $<3 \%$ of their body weight after $2-3$ months are typically considered non-responders. ${ }^{1,2}$ In prior randomized controlled trials of lifestyle interventions, non-response rates in the first 2 months have been reported for up to one third of actively 
treated participants, and observational data suggest that rates of non-response to lifestyle interventions may be even higher in real-world clinical practice. ${ }^{1,3,4}$ This is a high rate of failure for the first-line treatment approach for a high-morbidity disease that impacts more than $40 \%$ of American adults. ${ }^{5}$

The response to behavioural obesity treatment is likely determined by several factors that include a complex interplay of genetics, physiology, environment and individual behaviours. ${ }^{6}$ Ultimately, the patient has to reduce energy intake-the cornerstone of lifestyle intervention and driving force for achieving weight loss. Aside from the patient's individual characteristics, the ability to effectively reduce intake also depends on the treatment strategy being used. Use of total diet replacement (TDR) can regiment energy intake and reduce potential variation from the prescribed treatment due to simplicity and stimuli narrowing. ${ }^{7}$ A more consistent reduction in calorie intake using TDR could therefore increase the proportion of people who respond to treatment compared with food-based (FB), calorie-restricted diets.

Previously, primary outcome results from the OPTIWIN study were reported. OPTIWIN is the largest randomized controlled clinical trial of a TDR treatment programme conducted to date in the United States. ${ }^{8}$ OPTIWIN demonstrated that, compared with a comprehensive FB behavioural intervention, a TDR programme (the OPTIFAST programme [OP]) significantly increased the magnitude of weight loss at 6 and 12 months. After 12 months of treatment, there was a mean weight change of $-10.5 \%$ (SE $0.6 \%$ ) in the OP group versus $-5.5 \%$ (SE 0.6\%) in the FB group (difference $-4.9 \pm 0.9 \%, p<0.0001$ ). ${ }^{8}$ However, the mean weight change by treatment assignment does not provide a clear sense of what proportion of the population actually had a meaningful response to the assigned treatment. The OPTIWIN trial provides a unique opportunity to look at responsiveness to two behavioural interventions for weight reduction.

For this exploratory analysis, the primary research questions were as follows. (1) What percentage of people were responsive to their assigned treatment, both early and later in the course of treatment? (2) How was early treatment response related to longer term treatment response? Lastly, (3) what patient-level characteristics, within both treatment groups, were associated with response versus non-response? Treatment response was based on definitions from previous studies that showed that early weight loss thresholds of $<3 \%$ predicted long-term treatment non-response. ${ }^{1,2}$

\section{2 | METHODS}

This is a secondary data analysis of data from the OPTIWIN trial. The methods of the trial were published previously and are briefly described here. ${ }^{8}$

\section{1 | Setting and population}

OPTIWIN was an open-label, multicentre, randomized controlled clinical trial conducted by nine U.S. centres, including five private clinics and four academic medical centres. Participants were 18-70 years old, non-smokers, with a body mass index (BMI) of $30-55 \mathrm{~kg} / \mathrm{m}^{2}$. Subjects with recent weight loss or use of weight loss medications, previous bariatric surgery, organ failure, type 1 diabetes or haemoglobin $\mathrm{A} 1 \mathrm{c}>10 \%$, cardiovascular disease event or mental health hospitalization in the past 6 months, alcohol or drug dependence, positive screening for potential eating disorder, or poorly controlled depression were excluded. Following informed consent and an initial screening visit, eligible individuals completed a 7-day run-in diet that included use of one serving of meal replacement (MR) (OPTIFAST ${ }^{\circledR}$-Nestlé Health Science, Bridgewater, NJ) daily to assess tolerance to the product. Participants completed a final screening visit after the run-in to assess adherence to the run-in diet and self-monitoring tasks and to confirm final eligibility. This study was overseen and approved by a central institutional review board (quorum) after review by each site's institutional review board and registered on ClinicalTrials.gov (NCT02635698).

\section{2 | Treatment arms}

\subsection{1 | OPTIFAST programme}

The OP used TDR as the initial weight loss strategy for most participants. Number of MR recommended per day was based upon BMI, with the lowest amount being five servings, providing $800 \mathrm{kcal} /$ day. The MR provided $40 \%$ of calories as protein, $40 \%$ as carbohydrate and $20 \%$ as fat. Participants followed their prescription for 12 to 16 weeks based on provider discretion and patient preference, after which there was gradual reintroduction of food through week 26 . After week 26, participants' calories were gradually increased to achieve weight stability. During this time, participants were advised to use 1-2 MR daily to facilitate weight loss maintenance. All MR were provided at no cost.

Participants in the OP group were medically monitored according to the OPTIFAST protocol. This included clinic visits to address health concerns and adjust medications. Participants also had individual counselling with trained interventionists.

\subsection{2 | FB programme}

The FB was a modified version of the Diabetes Prevention Programme (DPP) intervention., 8 During active weight loss (0-26 weeks), FB participants reduced calories by lowering fat intake to $\leq 25 \%-30 \%$ of total calories. The goal was a reduction in calories of 500-750 kcal below estimated total energy expenditure, based on resting metabolic rate measured by indirect calorimetry (Microlife MedGem) plus an activity factor based on self-reported physical activity. The typical range of calories prescribed was $1200-1400 \mathrm{kcal} /$ day for women and $1600-1800 \mathrm{kcal} /$ day for men. FB participants received gift cards totaling $\$ 800$ to offset the cost of groceries during the active weight loss phase. They also had 
medical monitoring visits and individual counselling visits throughout the 52 weeks.

\subsection{3 | Comprehensive behavioural programme}

OP and FB groups had weekly, 45- to 60-min group behavioural sessions for the duration of the study, facilitated by trained professional interventionists using a standardized intervention manual. OP group sessions were based on the OPTIFAST Lifestyle Education Series, whereas FB programme used the DPP's Group Lifestyle Balance programme. ${ }^{9}$ A weekly target of $150-180$ min/week of moderate to vigorous exercise for all participants was recommended. All participants were also instructed to record daily food/beverage intake and minutes of exercise using written journals.

\section{3 | Study measures}

Body weight was measured in light clothing on calibrated scales to the nearest $0.1 \mathrm{lb}$. Height was measured without shoes using a wall-mounted stadiometer to the nearest $0.5 \mathrm{~cm}$. BMI was calculated as weight $(\mathrm{kg})$ divided by height $(\mathrm{m})$ squared. Waist circumference was measured using a spring-loaded tape measure (Gulick) $1 \mathrm{~cm}$ above the umbilicus. Blood pressure was measured in a seated position after a brief rest using automated blood pressure monitors. Two measures were averaged at each study visit.

Depressive symptoms and key dietary behaviours were measured using validated tools including the Current Major Depressive Disorder with Center for Epidemiological Studies depression Scale-Revised (CESD-R), The Eating Attitudes Test (EAT-26) and the Eating Inventory. ${ }^{10-12}$ These tools were scored for each subject for each instrument per published guidelines.

Metabolic risks including fasting blood glucose, haemoglobin A1c, insulin, total cholesterol, high-density lipoprotein (HDL) cholesterol, low-density lipoprotein (LDL) cholesterol and triglycerides were obtained from fasting blood samples obtained at each clinic visit (12, 26, 40 and 52 weeks) and analysed by a central laboratory. Metabolic syndrome was defined per the 'Diagnosis and Management of Metabolic Syndrome: An American Heart Association/National Heart, Lung and Blood Institute Scientific Statement (AHA/NHLBI Scientific Statement). ${ }^{13}$

Several process measures related to participant engagement in the interventions are also reported. Participants completed daily diaries of food and beverage intake and physical activity. Study interventionists compared the self-reported diaries with the study goals for diet and physical activity minutes on a weekly basis. The percent adherence was calculated by dividing the reported activity or intake by the study goal and multiplying by 100 (range $=0 \%-100 \%$ ). Attendance for all prescribed clinic visits and group behavioural sessions was tallied.

The primary outcome was a dichotomous variable for programme response versus non-response, defined as whether a participant achieved at least 3\% loss of initial body weight by 6 or 12 months of follow-up.

\section{4 | Analyses}

Analyses were primarily based on the modified intention-to-treat population, which included all randomized subjects known to have started the study programme and to have at least one post-baseline assessment of body weight. Missing values were imputed using last observation carried forward (LOCF). Sensitivity analyses used observed cases only rather than LOCF.

Baseline demographics and other characteristics were tabulated within groups of treatment arm and responder status. Differences in responder status within each treatment group for the baseline demographics and other characteristics were assessed using a chi-squared test for categorical measures and a standard $t$-test for continuous measures.

The probability of non-response at 3, 6, 9 and 12 months was calculated using counts only (crude model) and then modelled using a mixed logistic model (SAS/STAT Proc GLIMMIX) including adjustment for fixed effects measure of age, sex, race, initial BMI, diabetes status, previous weight loss attempts and Eating Inventory score with subject treated as a random effect (adjusted model). Predicted probabilities of non-response were calculated using the predicted probabilities from the mixed logistic model for each combination of treatment arm and time (3, 6, 9 and 12 months) as well as for covariates included in the models. Odds ratios (ORs) and 95\% confidence intervals (Cls) were also calculated from the mixed logistic model for categorical covariates. The probability of non-response conditional on response status at 3 months was calculated using counts only (crude model) due to small numbers within groups after conditioning on responder status at 3 months.

Means and standard deviations at baseline, follow-up and change over 12 months were calculated for several measures of cardiometabolic risk. $p$ values comparing the metabolic measures at baseline, at follow-up and for change from baseline to 12 months were computed from linear regression models with each individual metabolic measure as the outcome and fixed effects for responder status at 12 months, age, race, site and baseline diabetes status. Models for follow-up and change measures further included adjustment for the baseline measure. Similarly, baseline, follow-up and change values for each metabolic measure were compared between the OP responders and the FB responders using a linear regression model limited to responders with each individual metabolic measure as the outcome and fixed effects for treatment arm, age, race, site and baseline diabetes status. Adjustment for the baseline measure was further included in models for the follow-up and change measures. Process measures examining subject behaviours during the trial were tabulated within groups of treatment arm and responder status, and comparisons between all responders versus all non-responders as well as between responders in the OP group and responders in the FB group were made using standard $t$-tests. 
TAB LE 1 Baseline demographic and other characteristics of responders and non-responders at 12 months by treatment arm, $N$ (\%) or mean (SD)

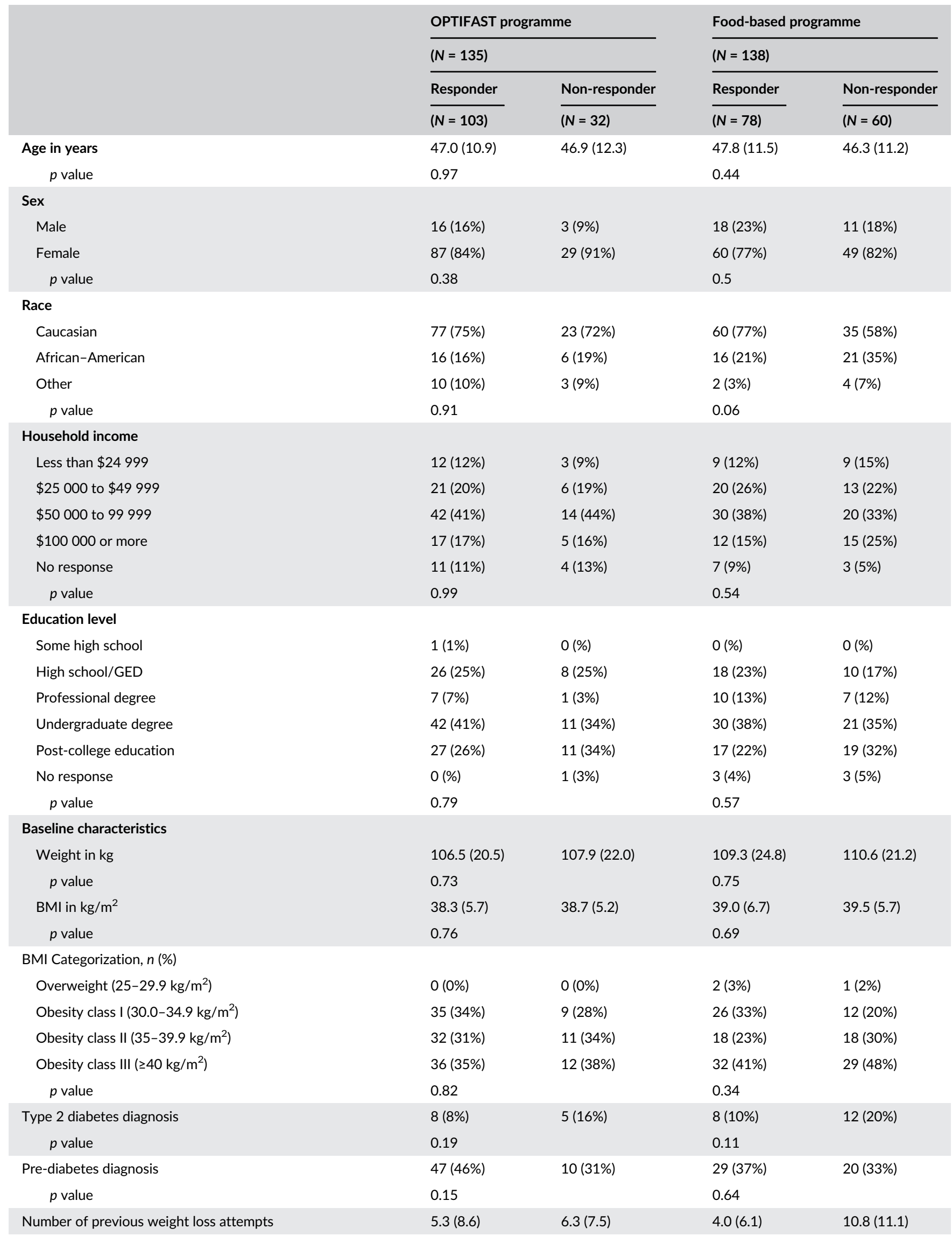


TABLE 1 (Continued)

\begin{tabular}{|c|c|c|c|c|}
\hline & \multicolumn{2}{|c|}{ OPTIFAST programme } & \multicolumn{2}{|c|}{ Food-based programme } \\
\hline & Responder & Non-responder & Responder & Non-responder \\
\hline & $(N=103)$ & $(N=32)$ & $(N=78)$ & $(N=60)$ \\
\hline$p$ value & 0.54 & & $<0.0001$ & \\
\hline$p$ value & 0.24 & & 0.15 & \\
\hline Used diabetes medications $^{a}$ & $8(8 \%)$ & $6(19 \%)$ & $8(10 \%)$ & $11(18 \%)$ \\
\hline$p$ value & 0.08 & & 0.17 & \\
\hline Used hypertension medications ${ }^{a}$ & 35 (34\%) & $11(34 \%)$ & $23(29 \%)$ & $20(33 \%)$ \\
\hline$p$ value & 0.97 & & 0.63 & \\
\hline$p$ value & 0.83 & & 0.79 & \\
\hline EAT-26 score & $6.7(5.1)$ & $6.8(4.4)$ & $5.6(4.0)$ & $8.1(5.5)$ \\
\hline$p$ value & 0.9 & & 0.003 & \\
\hline Eating Inventory: Total score & $24.1(6.4)$ & $22.6(5.9)$ & $21.8(7.2)$ & $24.0(7.3)$ \\
\hline$p$ value & 0.24 & & 0.08 & \\
\hline Eating Inventory: Dietary restraint & $10.5(4.1)$ & $9.9(4.1)$ & $10.1(4.7)$ & $10.3(4.7)$ \\
\hline$p$ value & 0.47 & & 0.8 & \\
\hline Eating Inventory: Disinhibition & $8.3(3.3)$ & 7.9 (3.5) & 7.4 (3.4) & $8.3(3.7)$ \\
\hline
\end{tabular}

Note. $p$ value comparing responder versus non-responder status within treatment groups from standard $t$-test for continuous variables or chi-squared test for categorical variables.

${ }^{a}$ Medication use includes use prior to study with continuation during study, or medication use that started during study and either continued throughout study or ended before study completion.

${ }^{\mathrm{b}} \mathrm{CESD}-\mathrm{R}=$ Current Major Depressive Disorder with Center for Epidemiological Studies Depression Scale-Revised.

All analyses were conducted using SAS/STAT software (Version 9.4 of The SAS System for Windows, SAS Institute, Cary, NC, USA).

\section{3 | RESULTS}

There were 103 (76\%) responders in the OP compared with 78 (57\%) in the FB group at 12 months $(p=0.0005)$. When comparing responders to non-responders within treatment groups (i.e., OP responder vs. OP non-responder), there were few differences in baseline characteristics (Table 1). For the FB group, Caucasians made up a higher proportion of the responder group (77\%), whereas the proportion of African-Americans in the non-responder group was higher (35\%); this result nearly reached statistical significance $(p=0.06)$. Non-responders in the FB group reported a significantly higher number of previous weight loss attempts $(+6.8, p<0.0001)$. The EAT-26 score was also significantly higher for non-responders in the FB group.
Figure 1 is a waterfall chart that displays each individual subject's weight change as a percentage (\%) at 12 months, by treatment group. The differing distributions of weight change values between the two groups show that the OP group had fewer participants with weight gain and more participants with higher percent of weight loss.

Predicted probabilities of non-response were derived from a mixed logistic regression with adjustment for sex, race, age, type 2 diabetes (T2D) at baseline, BMI category at baseline, previous weight loss attempts and Eating Inventory score. For the OP versus FB participants, the predicted probabilities of non-response at 3,6 , 9 and 12 months are shown in Figure 2.

The probability of being a non-responder at 12 months if a participant was a responder at 3 months is shown in Figure 3 using a crude model due to small numbers within groups after conditioning on responder status at 3 months. For FB participants who were responders at 3 months, there was a $12.8 \%$ chance that by 6 months, they would no longer be considered a responder. For OP participants, 


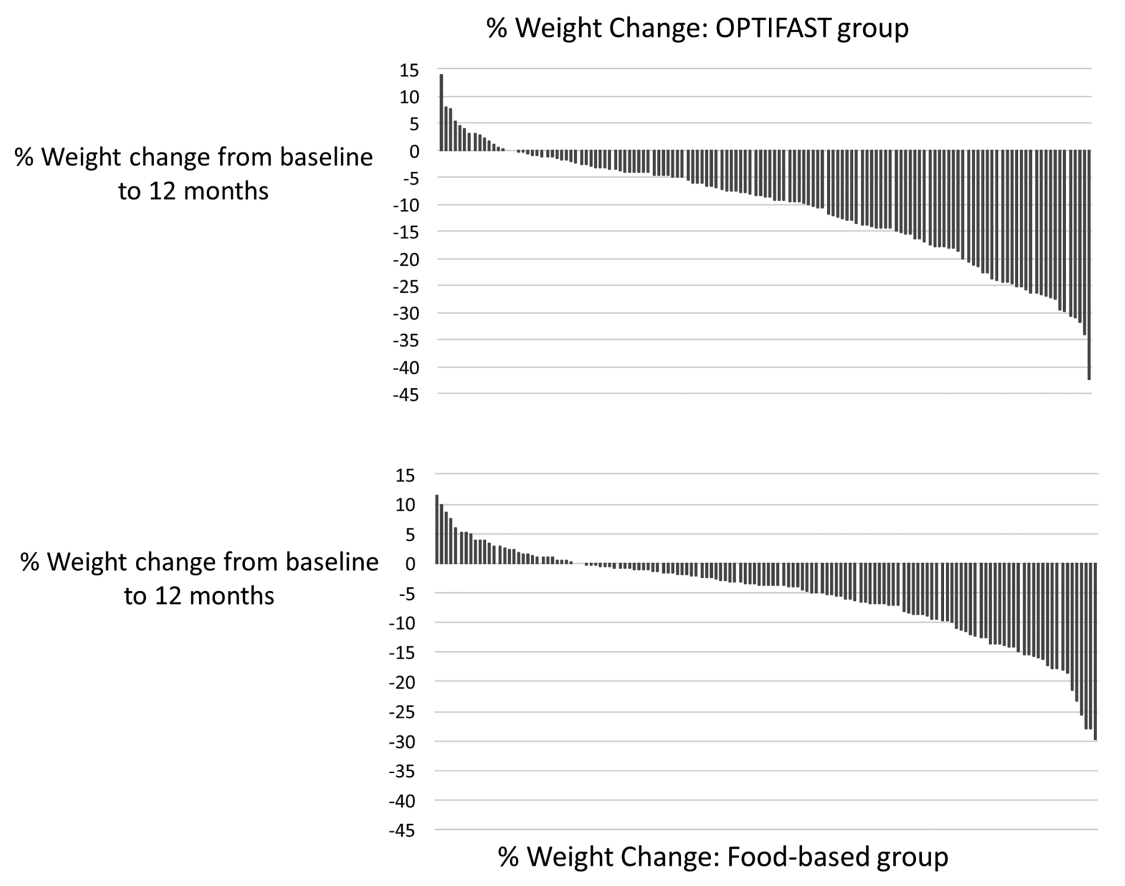

FIGURE 1 Waterfall chart showing weight change from baseline to 12 months for each individual participant ( $x$-axis) by treatment group

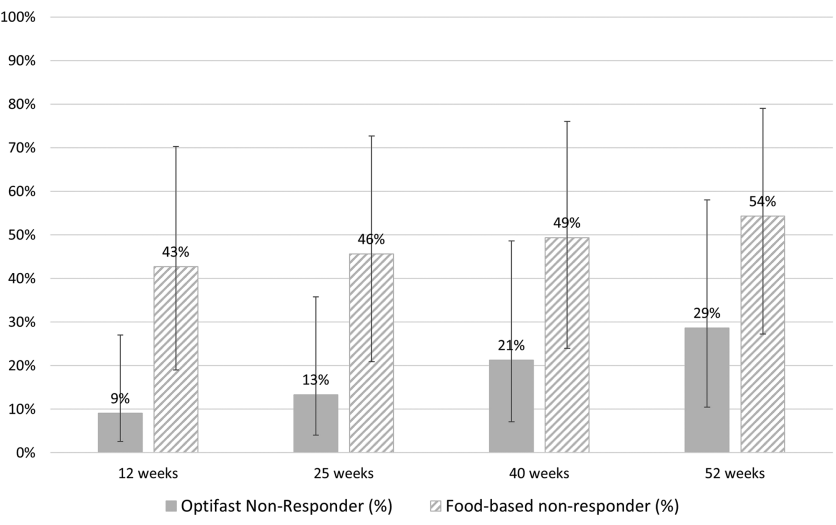

FIGURE 2 Predicted probabilities and 95\% confidence intervals for non-response by treatment group at each follow-up time point from mixed logistic regression model

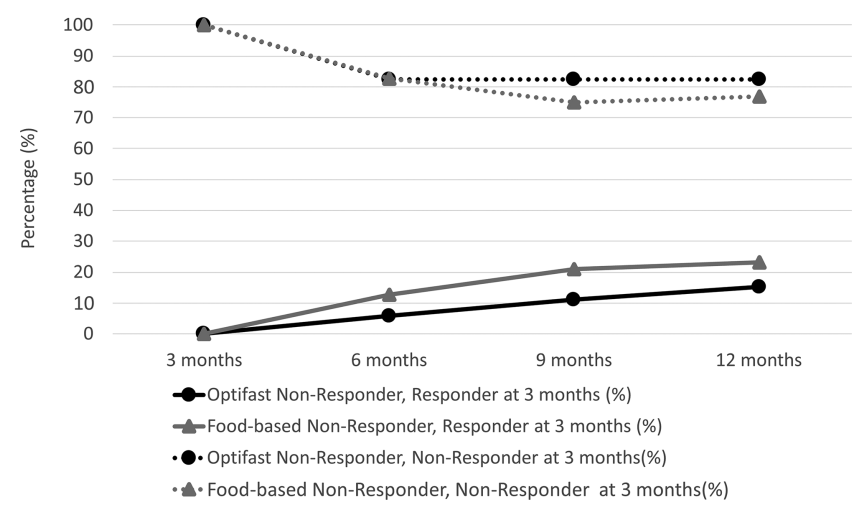

FIGURE 3 Crude probabilities of non-response based on response status at 3 months it was a $5.9 \%$ probability of the same outcome at 6 months. By 12 months, the probability for FB participants to be classified as non-responders if they had initially been a responder was $23.3 \%$ compared with $15.3 \%$ for OP participants. Conversely, those who were non-responders at 3 months had a high probability of staying a non-responder regardless of treatment assignment. From months 6-12, the probability for FB participants to remain non-responders was $76.9 \%$ and $82.4 \%$ for OP participants.

In the mixed logistic regression model with responder status as the outcome measure, race, T2D diabetes status and previous weight loss attempts were all significantly associated with responder status. The predicted probability of non-response among African-Americans was $40 \%$ compared with $17 \%$ in Caucasians (OR $=2.26,95 \%$ $\mathrm{Cl}=1.30-3.93, p=0.0075)$. Individuals without T2D were less likely to be non-responders than those with T2D ( $21 \%$ vs. $42 \%$, OR $=0.47$ $95 \% \mathrm{Cl}=0.23-0.97, p=0.046$ ). Previous weight loss attempts were significantly associated with responder status; increasing number of previous weight loss attempts was associated with increased odds of being a non-responder $(p=0.0023)$. Age approached significance with increasing age being associated with decreasing odds of being a non-responder ( $p=0.071$ ).

Changes in cardiometabolic risk factors are shown in Table 2. When non-responders of both treatment arms were compared with all responders, responders had significantly greater improvements in all markers except total and LDL-cholesterol $(p<0.01)$. There was also a significant decrease in the prevalence of metabolic syndrome within the responder groups for both treatment arms; the prevalence of metabolic syndrome decreased by approximately 50\% $(p<0.01)$. When comparing OP responders to $\mathrm{FB}$ responders, there were significantly greater improvements in waist circumference, HDL-cholesterol and triglycerides for the OP responders. 
TAB LE 2 Metabolic changes of responders and non-responders at 12 months by treatment arm, mean (SD) unless otherwise noted

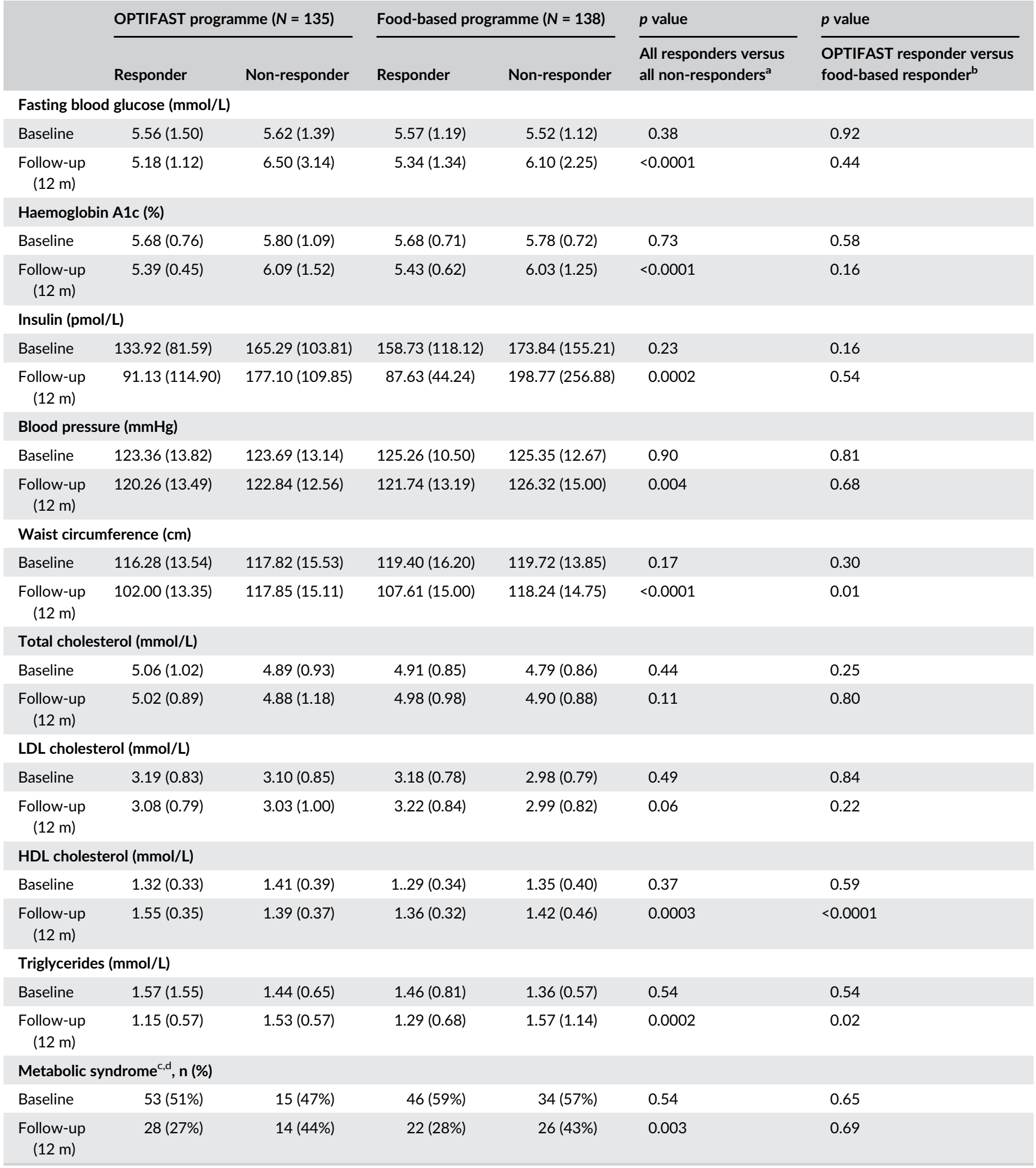

Note. Change from baseline is defined as 12 -month value - baseline value.

${ }^{a} p$ value comparing responders to non-responders from linear regression model with metabolic measure as the outcome and fixed effects for responder status at 52 weeks, age, race, site and baseline diabetes status. Models for follow-up and change further include adjustment for the baseline measure. ${ }^{\mathrm{b}} p$ value comparing OPTIFAST responders to food-based responders obtained from linear model limited to responders with metabolic measure as the outcome and fixed effects for treatment arm, age, race, site and baseline diabetes status. Models for follow-up and change further include adjustment for the baseline measure.

${ }^{c}$ Metabolic syndrome definition per 'Diagnosis and Management of Metabolic Syndrome: An American Heart Association/National Heart, Lung and Blood Institute Scientific Statement (AHA/NHLBI Scientific Statement). ${ }^{13}$

${ }^{\mathrm{d}}$ Metabolic syndrome $p$ values derived from logistic regression models with same parameterization as in footnotes a and $\mathrm{b}$. 
TABLE 3 Process measures among responders and non-responders at 12 months by treatment arm, mean (SD) unless otherwise noted

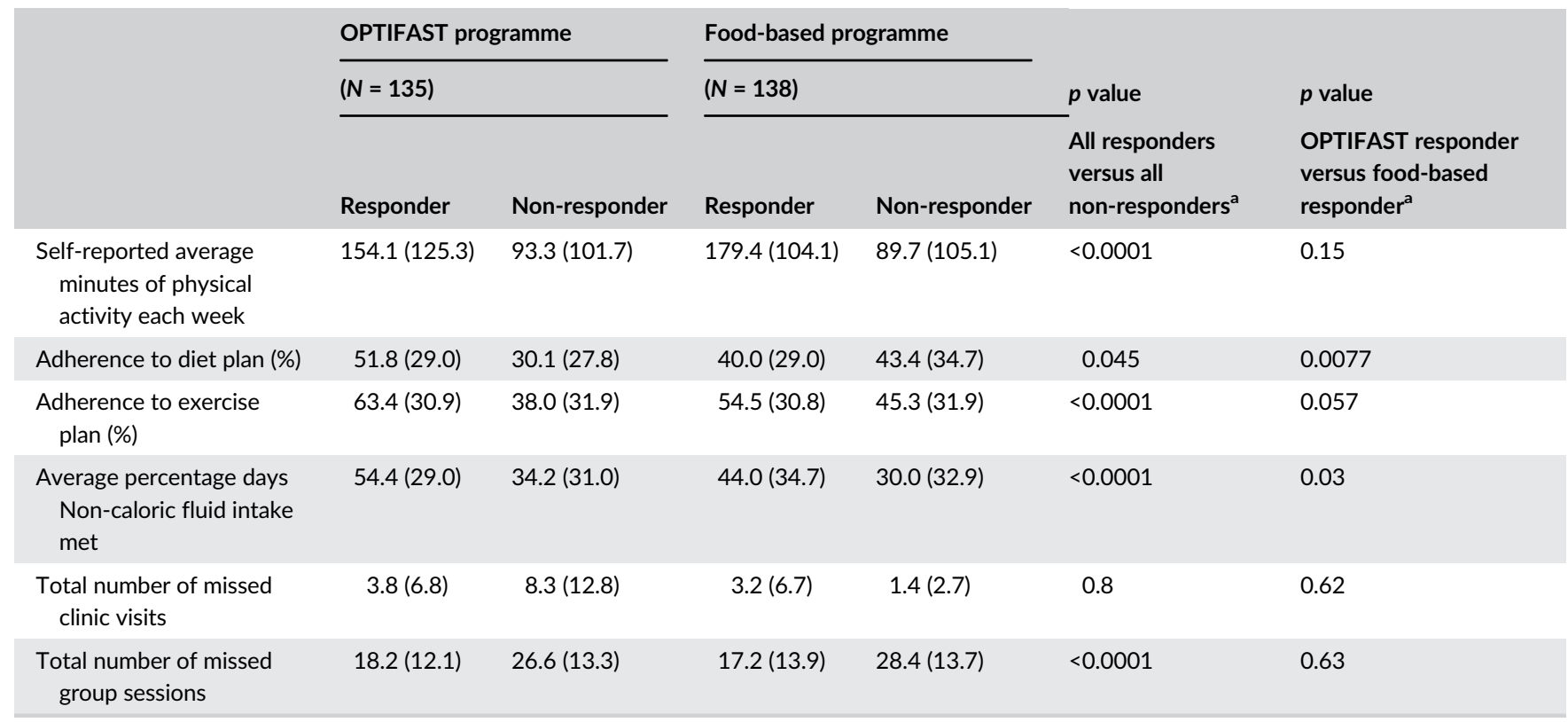

Note. For physical activity, adherence to diet and exercise plan and non-caloric fluid intake met, average is taken over all available measures for each subject over the study period. For total number of missed clinic visits and groups sessions, the value is cumulative for each subject over the study period. ${ }^{a} p$ values from standard $t$-test comparing responder versus non-responder status within treatment groups for OPTIFAST responder to food-based responder.

To determine if responders were reporting different behaviours compared with non-responders, and whether this varied by treatment group, several process measures as shown in Table 3 were assessed. When grouped by response status, responders reported higher average minutes of moderate physical activity, greater adherence to the dietary plan and exercise plans, greater non-calorie fluid intake and missed fewer group behavioural sessions. OP responders had higher percent adherence to prescribed meal plan and non-caloric fluid intake compared with FB responders. For FB responders, percent adherence to the diet plan $(40.0 \pm 29 \%)$ was similar to that of FB nonresponders $(43.4 \pm 34.7 \%)$.

\section{4 | DISCUSSION}

This secondary analysis of the OPTIWIN trial showed that a higher proportion of participants responded (i.e., lost $\geq 3 \%$ of their initial weight) to the OP compared with FB. The proportion of responders declined slightly over time within each group. More importantly, those who were not responders after 3 months of treatment were unlikely to be responders at 12 months, and as a result, they did not have improvements in cardiometabolic risk factors. The average adherence to the treatment meal plan and non-calorie fluid intake was higher for OP responders compared with FB responders. Indicators of higher probability of treatment non-response included African-American race, increasing numbers of weight loss attempts and T2D. The implications of this research are that even though the mean weight loss across the FB group was clinically significant, a significant proportion of people did not respond to a gold standard behavioural intervention for weight loss. By contrast, the OP treatment strategy leads to a higher rate of treatment response, thereby increasing the impact of the same type of behavioural weight loss intervention.

For obesity treatment, there is some tacit expectation that patients will learn their way out of the condition, and given a little more time and individual effort, a poor response to initial treatment will change for the better over time. Based on observations from clinical trials of anti-obesity pharmacotherapy, the Food and Drug Administration (FDA) has derived 'stopping rules' that identify people who are unlikely to have a response to treatment, typically by 12 weeks of treatment. ${ }^{14}$ This analysis from the OPTIWIN trial suggests that those who do not have a treatment response to these two strategies for behavioural weight loss by 12 weeks are unlikely to achieve that response on average by 12 months. This finding has been observed in other trials like Look AHEAD, where $28 \%$ of participants in the intensive lifestyle intervention lost $<3 \%$ at 2 months and had a mean weight loss of $3.98 \pm 4.3 \%$ at 1 year. $^{2}$ This does not mean that treatment should be abandoned, but it may suggest that treatment should be intensified or switched to an alternative strategy. Stepped care, which involves intensifying treatment based on pre-determined weight goals at various time points, has been tested in behavioural weight loss. The largest such trial by Jakicic et al. did not see an improvement in weight loss outcomes with one such stepped care intervention. ${ }^{15}$ In this study, two servings of MR per day were introduced at Step 5 of six total steps in the progression of treatment. Future studies might examine how to intensify treatment using a broader range of options including anti-obesity medication and TDR, particularly if starting from the point of a 
high-intensity, comprehensive intervention similar to the FB intervention tested in this trial.

Ideally, pre-treatment characteristics could be assessed that might predict treatment response to a given behavioural treatment approach. Overall, there were few distinctions between responders and non-responders at baseline, except that FB non-responders had more weight loss attempts and higher EAT-26 scores. Individuals who identify as African-American have experienced lower mean weight loss in other weight loss interventions, and as noted in this trial, treatment non-response was higher for African-Americans compared with Whites. ${ }^{16}$ T2D status was also associated with higher odds of nonresponse. This is not to suggest, however, that T2D cannot be successfully treated with weight reduction interventions, including TDR as recently demonstrated in the DiRECT trial. ${ }^{17}$ The proportion of individuals with $\mathrm{T} 2 \mathrm{D}$ in this trial was relatively small and included a wide range of anti-diabetes treatments, including those on insulin. ${ }^{8}$

Other researchers have shown that there are distinct patterns of response to behavioural interventions for weight control, based on key behaviours or individual characteristics. Fitzpatrick et al. used latent class analysis to identify how participants in the PREMIER and WLM trials engaged in key behaviours. ${ }^{18}$ Bartfield et al. also used participants from the PREMIER trial to examine weight loss trajectories and how behaviours were associated with the observed patterns. ${ }^{19}$ Both studies demonstrated that early adoption of behaviour change and sustained engagement with those behaviours, such as food journaling and physical activity, were associated with long-term weight loss. Rothberg et al. observed that older age, lower BMI at programme entry, lack of baseline depression or anxiety symptoms and early weight loss (i.e., 2 weeks) were all associated with increased retention and long-term weight loss in a medically monitored weight loss clinic. ${ }^{20}$

This is one of the first studies to look at differences in key behaviours for two active treatments with substantially different approaches to the calorie-restricted diet. Although the calorie prescription was lower for the OP group than the FB group, adherence to the dietary plan was significantly higher for OP responders compared with FB responders. This study does not allow us to identify why OP responders reported greater achievement of study treatment goals. There are likely aspects of the interventions as well as individual characteristics that affected engagement. For the OP intervention, which featured TDR, intervention-related factors at play could include ease of implementation due to simplified planning, preparation and limited choice, and the impact of stimuli narrowing. ${ }^{7}$ There remains a need to understand how baseline characteristics like race, age, previous number and type of weight loss attempts, co-morbid conditions and eating behaviours/attitudes might interact with treatment strategy to affect response. Additional research is needed in this area to assist clinicians with treatment prescribing.

The conclusions from this analysis are limited by several study design considerations. The OPTIWIN trial was designed to test the effectiveness of a TDR programme compared with an FB intervention. As a result, there were differences in contact frequency based on the medical monitoring protocol for OP. This may have contributed to differences in response rates. Additionally, although some of the process measures related to patient engagement were objective data, like class or clinic attendance, some of the measures were self-reported. These self-reported measures of diet and physical activity are subject to reporting error; however, there is no reason to believe that there would be systematic differences in reporting between the two treatment groups.

In conclusion, the OPTIWIN trial provided a unique opportunity to examine treatment response rates to two comprehensive behavioural interventions for weight loss. The OP was associated with a higher rate of treatment response compared with the FB intervention, and in general, those who were non-responders through 12 weeks were likely to be non-responders at 12 months, regardless of treatment group. Additional research is needed to understand how to optimize the initial treatment approach based on baseline characteristics and how to best handle treatment non-response within the first 12 weeks of treatment.

\section{CLINICAL TRIAL REGISTRATION}

Clinical trials.gov \#NCT02635698.

\section{CONFLICT OF INTERESTS}

JDA and JL are consultants for Nestlé Healthcare Nutrition; JDA, KHL, AER and LM receive grant funding from Nestlé Health Science; and SP is an employee of Nestlé Health Science. All remaining authors have no relevant disclosures. Nestlé Healthcare Nutrition owns the OPTIFAST programme.

\section{ORCID}

Jamy D. Ard (D) https://orcid.org/0000-0002-1643-6795

Kristina H. Lewis (D) https://orcid.org/0000-0002-4921-7230

\section{REFERENCES}

1. Unick JL, Pellegrini CA, Demos KE, Dorfman L. Initial weight loss response as an indicator for providing early rescue efforts to improve long-term treatment outcomes. Curr Diab Rep. 2017;17:69-78.

2. Unick JL, Hogan PE, Neiberg RH, et al. Evaluation of early weight loss thresholds for identifying nonresponders to an intensive lifestyle intervention. Obesity. 2014;22:1608-1616.

3. Ard J. Obesity in the US: what is the best role for primary care? BMJ. 2015;350:g7846-7856.

4. Lewis $\mathrm{KH}$, Fischer $\mathrm{H}$, Ard J, et al. Safety and effectiveness of longerterm phentermine use: clinical outcomes from an electronic health record cohort. Obesity. 2019;27:591-602.

5. Hales CM, Carroll MD, Fryar CD, Ogden CL. Prevalence of obesity and severe obesity among Adults: United States, 2017-2018. NCHS Data Brief. 2020;1-8.

6. Wadden TA, Tronieri JS, Butryn ML. Lifestyle modification approaches for the treatment of obesity in adults. Am Psychol. 2020; 75:235-251.

7. Kahathuduwa CN, Davis T, O'Boyle M, et al. Effects of 3-week total meal replacement vs. typical food-based diet on human brain functional magnetic resonance imaging food-cue reactivity and functional connectivity in people with obesity. Appetite. 2018;120:431-441.

8. Ard JD, Lewis $\mathrm{KH}$, Rothberg A, et al. Effectiveness of a total meal replacement program (OPTIFAST program) on weight loss: results from the OPTIWIN study. Obesity. 2019;27:22-29. 
9. Diabetes Prevention Program Research Group. The Diabetes Prevention Program (DPP): description of lifestyle intervention. Diabetes Care. 2002;25:2165-2171.

10. Garner DM, Olmsted MP, Bohr Y, Garfinkel PE. The eating attitudes test: psychometric features and clinical correlates. Psychol Med. 1982; 12:871-878.

11. Maruish ME. In: Mahwah NJ, ed. The use of psychological testing for treatment planning and outcomes assessment. 3rd ed. Lawrence Erlbaum Associates; 2004.

12. Stunkard AJ, Messick S. The three-factor eating questionnaire to measure dietary restraint, disinhibition and hunger. J Psychosom Res. 1985;29:71-83.

13. Grundy SM, Cleeman JI, Daniels SR, et al. Diagnosis and management of the metabolic syndrome: an American Heart Association/National Heart, Lung, and Blood Institute Scientific Statement. Circulation. 2005;112:2735-2752.

14. Krentz AJ, Fujioka K, Hompesch M. Evolution of pharmacological obesity treatments: focus on adverse side-effect profiles. Diabetes Obes Metab. 2016;18:558-570.

15. Jakicic JM, Tate DF, Lang W, et al. Effect of a stepped-care intervention approach on weight loss in adults: a randomized clinical trial. JAMA. 2012;307:2617-2626.

16. Lewis $\mathrm{KH}$, Edwards-Hampton SA, Ard JD. Disparities in treatment uptake and outcomes of patients with obesity in the USA. Curr Obes Rep. 2016;5:282-290.
17. Lean MEJ, Leslie WS, Barnes AC, et al. Durability of a primary careled weight-management intervention for remission of type 2 diabetes: 2-year results of the DiRECT open-label, cluster-randomised trial. The Lancet Diab \& Endocri. 2019;7:344-355.

18. Fitzpatrick SL, Coughlin JW, Appel LJ, et al. Application of latent class analysis to identify behavioral patterns of response to behavioral lifestyle interventions in overweight and obese adults. Int J Behav Med. 2015;22:471-480.

19. Bartfield JK, Stevens VJ, Jerome GJ, et al. Behavioral transitions and weight change patterns within the PREMIER trial. Obesity. 2011;19: 1609-1615.

20. Rothberg AE, McEwen LN, Kraftson AT, et al. Factors associated with participant retention in a clinical, intensive, behavioral weight management program. BMC Obes. 2015;2(11)::1-7.

How to cite this article: Ard JD, Lewis KH, Cohen SS, et al. Differences in treatment response to a total diet replacement intervention versus a food-based intervention: A secondary analysis of the OPTIWIN trial. Obes Sci Pract. 2020;6:

605-614. https://doi.org/10.1002/osp4.444 